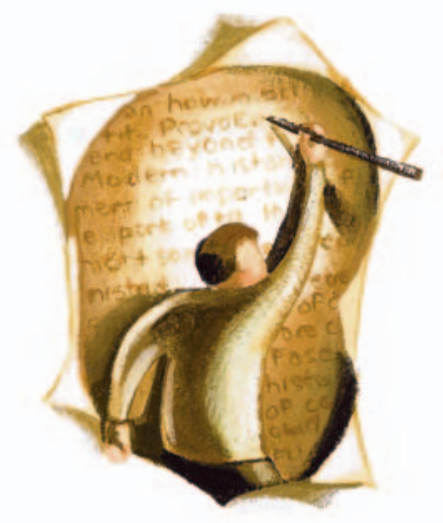

\section{Auricular acupuncture}

Neither the study by Taras Usichenko ${ }^{1}$ on auricular acupuncture for pain relief nor the accompanying commentary ${ }^{2}$ provide any information on the nature of the treatment that was being tested. One might be forgiven for assuming that auricular acupuncture is a form of traditional Chinese medicine, like body acupuncture. Few readers are probably aware that it was developed only in the I950s by a French physician, Paul Nogier. ${ }^{3}$ Although some experts believe that ear points were also used in traditional Chinese acupuncture, Nogier's auricular acupuncture is not based on traditional Chinese medical theory. Instead, an assumption that all internal organs are represented in the ear is the basis for auricular acupuncture. Therapists use maps that demonstrate these representations. One of the many problems with auricular acupuncture is that many such maps exist and little agreement exists regarding point location. Another problem is that all correspondence or reflex systems (e.g., auricular acupuncture, iridology and reflexology) fly in the face of our knowledge of anatomy and physiology.

\section{Edzard Ernst}

Professor of Complementary Medicine Peninsula Medical School

Exeter, UK

\section{REFERENCES}

I. Usichenko TI, Kuchling S, Witstruck T, et al. Auricular acupuncture for pain relief after ambulatory knee surgery: a randomized trial. CMAJ 2007;176 (2): $179-83$.
2. Buckley N. Auricular acupuncture for analgesia after arthroscopy [editorial]. CMAJ 2007;176(2):193-4 3. Nogier PMT. Handbook on auricular therapy. Moulins-les-Metz (France): Maisonneuve; I98I.

DOI:I0.1503/cmaj.1070008

\section{[Three of the authors respond:]}

We are grateful to Edzard Ernst for his comments concerning the origin and nature of auricular acupuncture, which we did not discuss in our recent article. ${ }^{1}$ The aim of our study was to test whether auricular acupuncture has analgesic properties, with groups of patients receiving acupuncture at specific acupuncture points or at nonacupuncture points (sham acupuncture). As we have now provided evidence for the effectiveness of auricular acupuncture ${ }^{1-3}$ we are planning to study the possible mechanisms underlying this therapy; it has been proposed that the endogenous opioid system plays a role. ${ }^{4}$

We mentioned the French origin of auricular acupuncture and our selection of Nogier's map of auricular points in our previous study, ${ }^{3}$ which we cited in our CMAJ article. One can certainly observe some parallels between auricular acupuncture, reflexology and iridology. However, when it comes to discussing potential mechanisms, we hesitate to compare auricular acupuncture, which has been shown to be effective in a number of animal studies and randomized clinical studies, ${ }^{1-4}$ with iridology, which is used only for diagnostic purposes and is considered to signal genotypes associated with certain medical conditions. ${ }^{5}$

\section{Taras I. Usichenko \\ Dragan Pavlovic \\ Michael Wendt}

Department of Anesthesiology and

Intensive Care Medicine

Ernst Moritz Arndt University

Greifswald, Germany

\section{REFERENCES}

I. Usichenko TI, Kuchling S, Witstruck T, et al. Auricular acupuncture for pain relief after ambulatory knee surgery: a randomized trial. CMAJ 2007;176 (2): $179-83$.
2. Usichenko TI, Dinse M, Hermsen M, et al. Auricular acupuncture for pain relief after total hip arthroplasty - a randomized controlled study. Pain 2005;II4:320-7.

3. Clement-Jones V, McLoughlin L, Tomlin S, et al. Increased beta-endorphin but not met-enkephalin levels in human cerebrospinal fluid after acupuncture for recurrent pain. Lancet I980;2(8201):946-9.

4. Asamoto S, Takeshige C. Activation of the satiety center by auricular acupuncture point stimulation. Brain Res Bull I992;29:157-64.

5. Um JY, An NH, Yang GB, et al. Novel approach of molecular genetic understanding of iridology: relationship between iris constitution and angiotensin converting enzyme gene polymorphism. Am JChin Med 2005;33:50I-5.

DOI:I0.I503/cmaj.I0700II

\section{Fundamental problem}

\section{with opioid trials for}

\section{chronic pain}

One problem with research on opioids for noncancer pain, in addition to those outlined by Andrea Furlan and associates, ${ }^{1}$ is the cognitive effect of these drugs, which makes it difficult to compare them with either placebo or active placebo such as NSAIDs. Even a nominal dose of opioids will improve mood through a euphoric effect. Tolerance develops such that the patient experiences intermittent withdrawal symptoms and smaller euphoric effects with trough and peak drug levels.

Under such conditions, patients will generally endorse the benefit of opioids for chronic noncancer pain. Euphoria is associated with pain relief and a greater sense of well-being, and the dysphoria and pain of withdrawal are associated with worsening chronic noncancer pain.

Further, it is often difficult for patients to recall how bad their pain was before they started treatment with opioids, and people taking opioids for chronic noncancer pain are likely to report an improvement in their overall condition even if they are worse off with the peak and trough effect of the opioid regimen. Perhaps they think that going without opioids altogether would feel like a trough: a state of withdrawal.

How else could research be done in 
this area? Delivering opioids via pumps that provide continuous infusions or fentanyl patches might overcome some of these problems. The dose would have to be titrated gradually, and a suitable placebo would be necessary. In trials comparing opioids in pill form with active placebo, perhaps the active placebo should contain one ingredient that gives nociceptive relief (e.g., NSAIDs) and a second ingredient that produces a calming but euphoric effect (e.g., a benzodiazepine such as lorazepam).

In traditional trials comparing oral opioids with placebo or NSAIDs, objective and detailed assessments of level of functioning and overall quality of life are better primary outcomes than pain. Participants' functioning, quality of life and pain should also be measured at a single point in time rather than being compared with baseline values.

\section{Tushar Mehta}

Substance Use Medical Service

St. Joseph's Health Centre

Toronto, Ont.

\section{REFERENCE}

I. Furlan AD, Sandoval JA, Mailis-Gagnon A, et al. Opioids for chronic noncancer pain: a metaanalysis of effectiveness and side effects. $C M A J$ 2006;I74(II):I589-94.

DOI:I0.I503/cmaj.ro6oI40

\section{[Three of the authors respond:]}

We thank Tushar Mehta for his comments regarding clinical trials to assess the efficacy of opioids for noncancer pain. Retrospective studies are indeed subject to recall bias, but we used only prospective controlled clinical trials in our meta-analysis. ${ }^{1}$ We extracted 2 outcome measures for our analyses: present pain intensity and present functional status. We avoided using outcomes measured as "better, the same or worse" because they are subject to recall bias. We were also careful to analyze the included studies with regard to valid outcome measures. The studies included in this review were judged to have valid outcome measures.

Mehta expresses doubts about the ability of a patient who is receiving opioids to validly report pain relief, but many studies have now established the validity of measures of pain relief as well as measures of functional status. Both of these types of outcome measures must be included if a trial is to be clinically relevant, and a valid prospective study must include comparisons of measurements taken at baseline and at subsequent points during treatment and follow-up in order to assess a therapy's efficacy.

In cases of substance abuse, opioids can be euphorigenic and continued use can lead to tolerance and even intermittent withdrawal symptoms. Our experience in pain relief clinics is that the great majority of people requiring opioids for pain relief are not addicted. Although it is normal for people to develop tolerance to opioids, nonaddicted patients with chronic noncancer pain do not commonly experience withdrawal phenomena if opioids are prescribed appropriately, and if sustainedrelease preparations are used.

\section{Andrea D. Furlan \\ Angela Mailis-Gagnon \\ Comprehensive Pain Program \\ Toronto Western Hospital \\ Toronto, Ont. \\ Eldon Tunks \\ Chedoke Rehabilitation Centre \\ Hamilton Health Sciences \\ Hamilton, Ont.}

\section{REFERENCE}

I. Furlan AD, Sandoval JA, Mailis-Gagnon A, et al. Opioids for chronic noncancer pain: a metaanalysis of effectiveness and side effects. $C M A J$ 2006;I74(II):I589-94.

DOI:I0.I503/cmaj.1070003

\section{Public involvement in guide-}

\section{line development}

Allan Detsky's timely editorial ${ }^{1}$ invites us to take a broader look at the biases that shape the recommendations in clinical practice guidelines, beyond financial links with the pharmaceutical industry. We strongly support Detsky's suggestion that nonexperts be included in guideline development panels, but we suggest that the value of public involvement should be judged not only on the basis of its impact on bias, but also in terms of democratic legitimacy and concordance with society's and patients' values.

Crafting recommendations involves making value judgments about the relative importance of competing goals and interests: maximizing health benefits for individuals and the population, promoting an equitable and effective allocation of resources, and respecting patients' autonomy. ${ }^{2}$ The crucial challenge is to ensure not only that recommendations are informed by the best available evidence, but also that the process is seen as transparent and legitimate. The quality of recommendations should be judged on the basis of their concordance with both the available evidence and the values of patients and society.

Patients are experts in their illness experience, life context and preferences. ${ }^{3}$ Like other experts, they have interests and are vulnerable to manipulation by the pharmaceutical industry. ${ }^{4}$ However, patient participation in guidelines committees could enhance the role of patients in clinical decision-making. ${ }^{5}$ Despite claims that they promote patient autonomy, clinical practice guidelines are rarely designed as instruments that facilitate a patient's choice.

Even if involvement by members of the general public in guideline development does not eliminate bias, at least their participation might bring a greater degree of legitimacy and accountability to the development process. Their participation would be in line with patients' desire to play a greater role in clinical decision-making.

\section{Antoine Boivin}

London School of Hygiene and

Tropical Medicine

London, UK

France Légaré

Associate Professor

Department of Family Medicine

Université Laval

Québec, Que.

\section{REFERENCES}

I. Detsky AS. Sources of bias for authors of clinical practice guidelines [editorial]. CMAJ 2006;175(9): I033.

2. Cohen J. Are clinical practice guidelines impartial? Int J Technol Assess Health Care 2004;20(4):415-20. 